PROCEEDINGS OF THE

AMERICAN MATHEMATICAL SOCIETY

Volume 131, Number 5, Pages 1423-1431

S 0002-9939(02)06676-5

Article electronically published on September 5, 2002

\title{
POSITIVE SOLUTIONS FOR A FOURTH ORDER EQUATION INVARIANT UNDER ISOMETRIES
}

\author{
FRÉDÉRIC ROBERT
}

(Communicated by Bennett Chow)

\begin{abstract}
Let $(M, g)$ be a smooth compact Riemannian manifold of dimension $n \geq 5$. We consider the problem

$$
\Delta_{g}^{2} u+\alpha \Delta_{g} u+a u=f u^{\frac{n+4}{n-4}}
$$

where $\Delta_{g}=-\operatorname{div}_{g}(\nabla), \alpha, a \in \mathbb{R}, u, f \in C^{\infty}(M)$. We require $u$ to be positive and invariant under isometries. We prove existence results for $(\star)$ on arbitrary compact manifolds. This includes the case of the geometric Paneitz-Branson operator on the sphere.
\end{abstract}

In 1983, Paneitz Pan introduced a fourth order operator defined on 4dimensional Riemannian manifolds. Branson Bra generalized the definition to $n$-dimensional Riemannian manifolds. Given $\left(M^{n}, g\right), n \geq 5$, a compact Riemannian manifold, and $u \in C^{\infty}\left(M^{n}\right)$, we let

$$
P_{g}^{n} u=\Delta_{g}^{2} u-d i v_{g}\left(a_{n} S_{g} g+b_{n} R i c_{g}\right) d u+\frac{n-4}{2} Q_{g}^{n} u .
$$

In this expression, $\Delta_{g} u=-d i v_{g}(\nabla u), S_{g}$ is the scalar curvature of $g, R i c_{g}$ its Ricci curvature, $a_{n}=\frac{(n-2)^{2}+4}{2(n-1)(n-2)}, b_{n}=-\frac{4}{n-2}$, and

$$
Q_{g}^{n}=\frac{1}{2(n-1)} \Delta_{g} S_{g}+\frac{n^{3}-4 n^{2}+16 n-16}{8(n-1)^{2}(n-2)^{2}} S_{g}^{2}-\frac{2}{(n-2)^{2}}\left|R i c_{g}\right|_{g}^{2} \text {. }
$$

If $\tilde{g}=\varphi^{4 /(n-4)} g$ is a conformal metric to $g$, then (see Branson [Bra])

$$
P_{g}^{n}(u \varphi)=\varphi^{\frac{n+4}{n-4}} P_{\tilde{g}}^{n}(u) \text { and } P_{g}^{n} \varphi=\frac{n-4}{2} Q_{\tilde{g}}^{n} \varphi^{\frac{n+4}{n-4}}
$$

where the first of these two equations holds for all smooth functions $u$ on $M^{n}$. Let $\left(\mathbb{S}^{n}, h\right)$ be the unit $n$-sphere. Then

$$
P_{h}^{n} u=\Delta_{h}^{2} u+c_{n} \Delta_{h} u+d_{n} u,
$$

where $c_{n}=\frac{n^{2}-2 n-4}{2}$ and $d_{n}=\frac{(n-4) n\left(n^{2}-4\right)}{16}$. We still refer to $P_{g}^{n}$ as the Paneitz operator. Given $\alpha, a \in \mathbb{R}$, let $P_{g}$ be the constant coefficient Paneitz type operator whose expression is $P_{g} u=\Delta_{g}^{2} u+\alpha \Delta_{g} u+a u$, where $u \in C^{\infty}\left(M^{n}\right)$. If $G$ is a group of isometries of $\left(M^{n}, g\right)$ and $f \in C^{\infty}(M)$ is invariant under the action of $G$, then

Received by the editors December 12, 2000 and, in revised form, December 7, 2001.

2000 Mathematics Subject Classification. Primary 35J35, 58J99.

(C)2002 American Mathematical Society 
we are interested in this paper in finding smooth positive $G$-invariant solutions of the fourth order equation

$$
P_{g} u=f u^{2^{\sharp}-1}
$$

where $2^{\sharp}=\frac{2 n}{n-4}$ is the critical Sobolev exponent for the embeddings of $H_{2}^{2}(M)$ in $L^{p}$-spaces. When $\left(M^{n}, g\right)$ is the unit $n$-sphere $\left(\mathbb{S}^{n}, h\right), \alpha=c_{n}$, and $a=d_{n}$, (1) reads as

$$
\Delta_{h}^{2} u+c_{n} \Delta_{h} u+d_{n} u=f u^{2^{\sharp}-1} .
$$

Then it follows from the above transformation laws that the existence of a smooth positive solution to (2) is equivalent to the existence of a conformal metric $g$ to $h$ such that $Q_{g}^{n}=f$. Equation (2) has its exact analogue when passing from the Paneitz operator to the conformal Laplacian on $\mathbb{S}^{n}, n \geq 3$. The equation associated to the conformal Laplacian reads as

$$
\Delta_{h} u+\frac{n(n-2)}{4} u=f u^{2^{\star}-1},
$$

where $2^{\star}=\frac{2 n}{n-2}$ and $f \in C^{\infty}(M)$, and we refer to the problem of finding smooth positive solutions to this equation as the Kazdan-Warner or the Nirenberg problem. Extending a result of Moser $\mathbb{M o s}$ from $\mathbb{S}^{2}$ to $\mathbb{S}^{3}$, Escobar and Schoen [EsSc] proved that if $f$ is a smooth positive function on $\mathbb{S}^{3}$, invariant under the action of a nontrivial group $G$ of isometries of $\left(\mathbb{S}^{3}, h\right)$ acting freely, then (3) possesses a smooth positive $G$-invariant solution. This result of Escobar and Schoen EsSc was then generalized by Hebey [Heb], where he proved that (3) still possesses a smooth positive $G$-invariant solution if we only require that the action of $G$ is without fixed points. A nontrivial group $G$ of isometries of a manifold $\left(M^{n}, g\right)$ is said to act freely if $M^{n} / G$ is still a manifold. We say that $G$ acts without fixed points if for any $x$, the $G$-orbit $O_{G}(x)$ of $x$ has at least two elements. A nontrivial group acting freely acts without fixed points. Returning to (2), it was proved in Djadli-Hebey-Ledoux DHL that if $f$ is a smooth positive function on $\mathbb{S}^{5}$, invariant under the action of a nontrivial group $G$ of isometries of $\left(\mathbb{S}^{5}, h\right)$ acting freely, then (2) possesses a smooth positive $G$-invariant solution. Hebey put to our attention the question of whether or not such a result holds when the condition that $G$ acts freely is replaced by the less restrictive condition that $G$ acts without fixed points. We answer this question by the affirmative, and prove the following theorem:

Theorem 1. Let $G$ be a compact subgroup of isometries of the standard sphere $\left(\mathbb{S}^{5}, h\right), f \in C^{\infty}\left(\mathbb{S}^{5}\right)$ positive and $G$-invariant. Assume that $G$ acts without fixed points. Then (2) possesses a smooth positive $G$-invariant solution, and there exists a conformal $G$-invariant metric $g$ to $h$ such that $Q_{g}^{5}=f$.

References where (11) and (2) are studied are Djadli-Hebey-Ledoux [DHL], HebeyRobert [HeRo], and Jourdain [Jou].

\section{The CASE of AN ARbitrary Riemannian manifold}

Let $\left(M^{n}, g\right)$ be a compact Riemannian manifold of dimension $n \geq 5$. Not to carry heavy notations, we note $M$ instead of $M^{n}$. If $\operatorname{Isom}_{g}(M)$ is the isometry group of $(M, g)$, we let $G$ be a compact subgroup of $I_{s o m}(M)$. Given $f \in C^{\infty}(M)$, positive 
and $G$-invariant, and given $a, \alpha>0$, we let

$$
\lambda^{G}(f)=\inf _{u \in \mathcal{H}_{f}^{G}} \int_{M}\left(\left(\Delta_{g} u\right)^{2}+\alpha|\nabla u|_{g}^{2}+a u^{2}\right) d v_{g},
$$

where $d v_{g}$ is the Riemannian volume element for $g$, and $\mathcal{H}_{f}^{G}$ is the set consisting of $G$-invariant functions in $H_{2}^{2}(M)$ which are such that $\int_{M} f|u|^{2^{\sharp}} d v_{g}=1$. It can be checked that whatever $(M, g)$ is, whatever $f$ is, and whatever $a$ and $\alpha$ are,

$$
\lambda^{G}(f) \leq \frac{\left|O_{G}(x)\right|^{\frac{4}{n}}}{K_{0} f(x)^{\frac{2}{2^{\sharp}}}}
$$

for all $x \in M$, where $\left|O_{G}(x)\right|$ is the cardinality of the orbit $O_{G}(x)$ and $K_{0}$ is the best constant for the optimal Sobolev Euclidean inequality

$$
\left(\int_{\mathbb{R}^{n}}|u|^{2^{\sharp}} d v_{\xi}\right)^{\frac{2}{2^{\sharp}}} \leq K_{0} \int_{\mathbb{R}^{n}}\left(\Delta_{\xi} u\right)^{2} d v_{\xi}
$$

where $d v_{\xi}$ is the volume element in $\mathbb{R}^{n}$ and $\Delta_{\xi}$ is the usual Laplacian with the minus sign convention. The first objective of this section is to prove the following theorem:

Theorem 2. Let $(M, g)$ be a compact Riemannian manifold of dimension $n \geq 5$, $G$ a compact subgroup of $I_{s o m}(M), f \in C^{\infty}(M)$, positive and $G$-invariant, and $a, \alpha>0$. If $a \leq \frac{\alpha^{2}}{4}$, and if for all $x \in M$,

$$
\lambda^{G}(f)<\frac{\left|O_{G}(x)\right|^{\frac{4}{n}}}{K_{0} f(x)^{\frac{2}{2 \sharp}}}
$$

then (11) possesses a smooth positive $G$-invariant solution.

We prove this theorem in what follows. For $0<\epsilon<2^{\sharp}-2$, we define

$$
\lambda_{\epsilon}^{G}(f)=\inf _{u \in \mathcal{H}_{f, \epsilon}^{G}}\left(\int_{M}\left(\left(\Delta_{g} u\right)^{2}+\alpha|\nabla u|_{g}^{2}+a u^{2}\right) d v_{g}\right)
$$

where $\mathcal{H}_{f, \epsilon}^{G}$ is the set consisting of $G$-invariant functions in $H_{2}^{2}(M)$ which are such that $\int_{M} f|u|^{2^{\sharp}-\epsilon} d v_{g}=1$. The following lemma easily follows from what has been achieved in DHL.

Lemma 1. Let $(M, g)$ be a compact Riemannian manifold of dimension $n \geq 5$. Let $G$ be a subgroup of Isom $(M), f \in C^{\infty}(M)$ a positive $G$-invariant function, and $a, \alpha>0$ such that $a \leq \frac{\alpha^{2}}{4}$. Then $\lambda_{\epsilon}^{G}(f)$ is attained by a smooth positive $G$-invariant function $u_{\epsilon}$ which satisfies

$$
\Delta_{g}^{2} u_{\epsilon}+\alpha \Delta_{g} u_{\epsilon}+a u_{\epsilon}=\lambda_{\epsilon}^{G}(f) f u_{\epsilon}^{2^{\sharp}-1-\epsilon}
$$

and $\int_{M} f u_{\epsilon}^{2^{\sharp}-\epsilon} d v_{g}=1$. Moreover, up to a subsequence, $\left(u_{\epsilon}\right)$ converges weakly in $H_{2}^{2}(M)$ to a function $u$. If $u \neq \equiv$, then $u$ is a positive smooth $G$-invariant function which realizes $\lambda^{G}(f)$, and, up to a positive constant scale factor, $u$ is a solution of (11).

We proceed with the proof of Theorem 2. We assume that (6) is true. We let $\left(u_{\epsilon}\right)$ be the sequence of Lemma 1. Also let $\lambda=\limsup \lambda_{\epsilon}^{G}(f)$. Then $\lambda \leq \lambda^{G}(f)$, and with Hölder and Sobolev inequalities we get that $\lambda>0$. Assume now that there is no positive $G$-invariant solution $u \in C^{\infty}(M)$ to (11). Then $u_{\epsilon} \rightarrow 0$ almost everywhere. 
Let $x_{\epsilon} \in M$ be such that $u_{\epsilon}\left(x_{\epsilon}\right)=\sup _{M} u_{\epsilon}$. If $u_{\epsilon}\left(x_{\epsilon}\right)$ is bounded, it follows from classical regularity theory (see for instance [GT] ) that $\left(u_{\epsilon}\right)$ is bounded in $C^{4, \beta}(M)$, $0<\beta<1$. Then $u_{\epsilon} \rightarrow 0$ in $C^{4}(M)$, a contradiction since $\int_{M} f u_{\epsilon}^{2^{\sharp}-\epsilon} d v_{g}=1$. Hence, $u_{\epsilon}\left(x_{\epsilon}\right) \rightarrow+\infty$. Let $x_{1} \in M$ be such that $x_{\epsilon} \rightarrow x_{1}$. We define $\mu_{\epsilon}=u_{\epsilon}\left(x_{\epsilon}\right)^{-\frac{2}{n-4}}$ and $k_{\epsilon}=\mu_{\epsilon}^{1-\epsilon \frac{n-4}{8}}$. For $|x|<\frac{i_{g}(M)}{k_{\epsilon}}$, where $i_{g}(M)>0$ is the injectivity radius of $M$, we let

$$
v_{\epsilon}(x)=\mu_{\epsilon}^{\frac{n-4}{2}} u_{\epsilon}\left(\exp _{x_{\epsilon}}\left(k_{\epsilon} x\right)\right) \text { and } g_{\epsilon}=\left(\exp _{x_{\epsilon}}^{\star} g\right)\left(k_{\epsilon} x\right),
$$

where $\exp _{x_{\epsilon}}$ denotes the exponential map at $x_{\epsilon}$. Then $v_{\epsilon}$ verifies

$$
\Delta_{g_{\epsilon}}^{2} v_{\epsilon}+\alpha k_{\epsilon}^{2} \Delta_{g_{\epsilon}} v_{\epsilon}+a k_{\epsilon}^{4} v_{\epsilon}=\lambda_{\epsilon}^{G}(f) f\left(\exp _{x_{\epsilon}}\left(k_{\epsilon} x\right)\right) v_{\epsilon}^{2^{\sharp}-1-\epsilon},
$$

an equation which can also be read as

$$
\left(\Delta_{g_{\epsilon}}+\frac{\alpha k_{\epsilon}^{2}}{2}\right)^{2} v_{\epsilon}=\lambda_{\epsilon}^{G}(f) f\left(\exp _{x_{\epsilon}}\left(k_{\epsilon} x\right)\right) v_{\epsilon}^{2^{\sharp}-1-\epsilon}+\left(\frac{\alpha^{2}}{4}-a\right) k_{\epsilon}^{4} v_{\epsilon} .
$$

We have $0 \leq v_{\epsilon} \leq 1$ and $k_{\epsilon} \rightarrow 0$. By classical regularity theorems (see for instance GT] $),\left(v_{\epsilon}\right)$ is bounded in $C^{4, \beta}(K)$ for $0<\beta<1$ and all compact subsets $K \subset \mathbb{R}^{n}$. Then, up to a subsequence, there exists $v \in C^{4}\left(\mathbb{R}^{n}\right)$ such that $v_{\epsilon}$ goes to $v$ in $C_{l o c}^{4}\left(\mathbb{R}^{n}\right)$. In particular $v \geq 0, v(0)=1$, and

$$
\Delta_{\xi}^{2} v=\lambda f\left(x_{1}\right) v^{2^{\sharp}-1} .
$$

Then (see [HeRo]) we know precisely what $v$ is. Given $x \in M$ and $r>0$, we let $B_{g}(x, r)$ be the geodesic ball of center $x$ and radius $r$ in $M$, and for $p \in \mathbb{R}^{n}$, we let $B_{\xi}(p, r)$ be the Euclidean ball in $\mathbb{R}^{n}$ of center $p$ and radius $r$. For $R>0$, we have

$$
\begin{aligned}
\int_{B_{g}\left(x_{\epsilon}, R k_{\epsilon}\right)} f u_{\epsilon}^{2^{\sharp}-\epsilon} d v_{g} & =\left(\mu_{\epsilon}^{-1}\right)^{\epsilon \frac{(n-4)^{2}}{8}} \int_{B_{\xi}(0, R)} f\left(\exp _{x_{\epsilon}}\left(k_{\epsilon} x\right)\right) v_{\epsilon}^{2^{\sharp}-\epsilon} d v_{g_{\epsilon}} \\
& \geq f\left(x_{1}\right) \int_{B_{\xi}(0, R)} v^{2^{\sharp}} d v_{\xi}+o(1)
\end{aligned}
$$

since $\mu_{\epsilon} \rightarrow 0$ and $v_{\epsilon} \rightarrow v$ in $C^{4}\left(B_{\xi}(0, R)\right)$. Now, since we also have that $x_{\epsilon} \rightarrow x_{1}$, $k_{\epsilon} \rightarrow 0$ and $f \geq 0$, we obtain that for any $\delta>0$,

$$
\int_{B_{g}\left(x_{1}, \delta\right)} f u_{\epsilon}^{2^{\sharp}-\epsilon} d v_{g} \geq f\left(x_{1}\right) \int_{\mathbb{R}^{n}} v^{2^{\sharp}} d v_{\xi}+o(1) .
$$

Let $O_{G}\left(x_{1}\right)=\left\{x_{1}, \ldots, x_{m}\right\}$. Since $f$ is $G$-invariant and $G$ is a group of isometries,

$$
\int_{B_{g}\left(x_{i}, \delta\right)} f u_{\epsilon}^{2^{\sharp}-\epsilon} d v_{g}=\int_{B_{g}\left(x_{1}, \delta\right)} f u_{\epsilon}^{2^{\sharp}-\epsilon} d v_{g} \geq f\left(x_{1}\right) \int_{\mathbb{R}^{n}} v^{2^{\sharp}} d v_{\xi}+o(1)
$$

for all $i=1, \ldots, m$. Taking $\delta>0$ sufficiently small, we obtain

$$
1=\int_{M} f u_{\epsilon}^{2^{\sharp}-\epsilon} d v_{g} \geq m f\left(x_{1}\right) \int_{\mathbb{R}^{n}} v^{2^{\sharp}} d v_{\xi}+o(1) .
$$

Multiplying by $v$ the equation satisfied by $v$, and integrating, it follows with (5), (4), and the inequality $\lambda \leq \lambda^{G}(f)$, that $v$ is minimizing for (51) and that

$$
\lambda^{G}(f)=\lambda=\frac{\left|O_{G}\left(x_{1}\right)\right|^{\frac{4}{n}}}{f\left(x_{1}\right)^{\frac{2}{2^{\sharp}}} K_{0}} .
$$

A contradiction with ([6). This proves Theorem 2 . 
We proceed in what follows with the study of the behaviour of the $u_{\epsilon}$ 's. We assume as in the proof of Theorem 2 that $u_{\epsilon} \rightarrow 0$ almost everywhere. It follows from the proof of Theorem 2 that equality holds in (9). Then, for any $\delta$ small,

$$
\int_{B_{g}\left(x_{1}, \delta\right)} f u_{\epsilon}^{2^{\sharp}-\epsilon} d v_{g}=\frac{1}{\left|O_{G}\left(x_{1}\right)\right|}+o(1) .
$$

We also get that $\mu_{\epsilon}^{\epsilon} \rightarrow 1$ and that for any $\Omega \subset \subset M \backslash O_{G}\left(x_{1}\right)$,

$$
\int_{\Omega} u_{\epsilon}^{2^{\sharp}-\epsilon} d v_{g}=o(1) .
$$

We now give a more precise description of the convergence of $\left(u_{\epsilon}\right)$ outside the orbit $O_{G}\left(x_{1}\right)$. Let $\sigma_{1}=I d_{M}, \sigma_{2}, \ldots, \sigma_{m} \in G$ be such that $x_{i}=\sigma_{i}\left(x_{1}\right)$ where $O_{G}\left(x_{1}\right)=\left\{x_{1}, \ldots, x_{m}\right\}$. Define $x_{\epsilon, i}=\sigma_{i}\left(x_{\epsilon}\right)$. First, we want to prove that there exists $C>0$ such that for any $x \in M$,

$$
\inf _{i=1, \ldots, p} d_{g}\left(x, x_{\epsilon, i}\right)^{\frac{4(n-4)}{8-\epsilon(n-4)}} u_{\epsilon}(x) \leq C .
$$

We follow an idea of Druet [Dru]. Assume that there exists $y_{\epsilon} \in M$ such that

$$
\sup _{x \in M} \inf _{i=1, \ldots, p} d_{g}\left(x, x_{\epsilon, i}\right)^{s_{\epsilon}} u_{\epsilon}(x)=\inf _{i=1, \ldots, p} d_{g}\left(y_{\epsilon}, x_{\epsilon, i}\right)^{s_{\epsilon}} u_{\epsilon}\left(y_{\epsilon}\right) \rightarrow+\infty
$$

where $s_{\epsilon}=\frac{4(n-4)}{8-\epsilon(n-4)}$. Define $\hat{\mu}_{\epsilon}=u_{\epsilon}\left(y_{\epsilon}\right)^{-\frac{2}{n-4}}, \hat{k}_{\epsilon}=\hat{\mu}_{\epsilon}^{1-\epsilon \frac{n-4}{8}}$, and set

$$
\hat{v}_{\epsilon}(x)=\hat{\mu}_{\epsilon}^{\frac{n-4}{2}} u_{\epsilon}\left(\exp _{y_{\epsilon}}\left(\hat{k}_{\epsilon} x\right)\right)
$$

For $|x|<\frac{i_{g}(M)}{\hat{k}_{\epsilon}}$ and $\hat{g}_{\epsilon}(x)=\exp _{y_{\epsilon}}^{\star} g\left(\hat{k}_{\epsilon} x\right)$, we have

$$
\Delta_{\hat{g}_{\epsilon}}^{2} \hat{v}_{\epsilon}+\alpha k_{\epsilon}^{2} \Delta_{\hat{g}_{\epsilon}} \hat{v}_{\epsilon}+a k_{\epsilon}^{4} \hat{v}_{\epsilon}=f\left(\exp _{y_{\epsilon}}\left(\hat{k}_{\epsilon} x\right)\right) \hat{v}_{\epsilon}^{2^{\sharp}-1-\epsilon} .
$$

Let $R>0$. With (14) and $|x| \leq R$, we obtain

$$
\hat{v}_{\epsilon}(x)=\frac{u_{\epsilon}\left(\exp _{y_{\epsilon}}\left(\hat{k}_{\epsilon} x\right)\right)}{u_{\epsilon}\left(y_{\epsilon}\right)} \leq\left(\frac{\inf _{i=1, \ldots, p} d_{g}\left(y_{\epsilon}, x_{\epsilon, i}\right)}{\inf _{i=1, \ldots, p} d_{g}\left(\exp _{y_{\epsilon}}\left(\hat{k}_{\epsilon} x\right), x_{\epsilon, i}\right)}\right)^{\frac{4(n-4)}{8-\epsilon(n-4)}} .
$$

Since $\inf _{i=1, \ldots, p} d_{g}\left(\exp _{y_{\epsilon}}\left(\hat{k}_{\epsilon} x\right), x_{\epsilon, i}\right) \geq \inf _{i=1, \ldots, p} d_{g}\left(y_{\epsilon}, x_{\epsilon, i}\right)-\hat{k}_{\epsilon} R$,

$$
\hat{v}_{\epsilon}(x) \leq\left(1-R \frac{\hat{k}_{\epsilon}}{\inf _{i=1, \ldots, p} d_{g}\left(y_{\epsilon}, x_{\epsilon, i}\right)}\right)^{-\frac{4(n-4)}{8-\epsilon(n-4)}}
$$

for all $|x| \leq R$. Now, with (14), we obtain that

$$
\frac{\inf _{i=1, \ldots, p} d_{g}\left(y_{\epsilon}, x_{\epsilon, i}\right)}{\hat{k}_{\epsilon}} \rightarrow+\infty
$$

Then $\hat{v}_{\epsilon}$ is uniformly bounded on every compact set. Writing that

$$
\left(\Delta_{\hat{g}_{\epsilon}}+\frac{\alpha \hat{k}_{\epsilon}^{2}}{2}\right)^{2} \hat{v}_{\epsilon}=f\left(\exp _{y_{\epsilon}}\left(\hat{k}_{\epsilon} x\right)\right) \hat{v}_{\epsilon}^{2^{\sharp}-1-\epsilon}+\left(\frac{\alpha^{2}}{4}-a\right) \hat{k}_{\epsilon}^{4} \hat{v}_{\epsilon}
$$


and using classical regularity results (see for instance [GT]), there exists $\hat{v} \in C^{4}\left(\mathbb{R}^{n}\right)$ such that, up to a subsequence, $\hat{v}_{\epsilon} \rightarrow \hat{v}$ in $C_{l o c}^{4}\left(\mathbb{R}^{n}\right)$, and $\hat{v}(0)=1$. Now, as is easily checked,

$$
\int_{B_{g}\left(y_{\epsilon}, \hat{k}_{\epsilon}\right)} u_{\epsilon}^{2^{\sharp}-\epsilon} d v_{g}=\hat{\mu}_{\epsilon}^{-\epsilon \frac{(n-4)^{2}}{8}} \int_{B_{\xi}(0,1)} \hat{v}_{\epsilon}^{2^{\sharp}-\epsilon} d v_{\hat{g}_{\epsilon}} .
$$

Since $\hat{\mu}_{\epsilon} \leq 1$, when $\epsilon \rightarrow 0$, then

$$
\int_{B_{g}\left(y_{\epsilon}, \hat{k}_{\epsilon}\right)} u_{\epsilon}^{2^{\sharp}-\epsilon} d v_{g} \geq \int_{B_{\xi}(0,1)} \hat{v}^{2^{\sharp}} d v_{\xi}+o(1) .
$$

Now, up to a subsequence, we can assume that $y_{\epsilon} \rightarrow y_{0} \in M$. If $y_{0} \notin O_{G}\left(x_{1}\right)$, then, with (12), we get that $\int_{B_{g}\left(y_{\epsilon}, \hat{k}_{\epsilon}\right)} u_{\epsilon}^{2^{\sharp}-\epsilon} d v_{g} \rightarrow 0$. Then $\int_{B_{\xi}(0,1)} \hat{v}^{2^{\sharp}} d v_{\xi}=0$, a contradiction. Hence, up to an isometry of $G$, we can assume that $y_{0}=x_{1}$. Taking $\delta>0$ small enough,

$$
\int_{B_{g}\left(y_{\epsilon}, \hat{k}_{\epsilon}\right)} u_{\epsilon}^{2^{\sharp}-\epsilon} d v_{g}=\int_{B_{g}\left(y_{\epsilon}, \hat{k}_{\epsilon}\right) \cap B_{g}\left(x_{1}, \delta\right)} u_{\epsilon}^{2^{\sharp}-\epsilon} d v_{g} .
$$

For any $R^{\prime}>0$, we have

$$
\int_{B_{g}\left(x_{1}, \delta\right) \backslash B_{g}\left(x_{\epsilon}, R^{\prime} k_{\epsilon}\right)} u_{\epsilon}^{2^{\sharp}-\epsilon} d v_{g} \leq \epsilon\left(R^{\prime}\right)+o(1)
$$

where $\lim _{R^{\prime} \rightarrow+\infty} \epsilon\left(R^{\prime}\right)=0$. It follows that

$$
\int_{B_{g}\left(y_{\epsilon}, \hat{k}_{\epsilon}\right)} u_{\epsilon}^{2^{\sharp}-\epsilon} d v_{g} \leq \int_{B_{g}\left(y_{\epsilon}, \hat{k}_{\epsilon}\right) \cap B_{g}\left(x_{\epsilon}, R^{\prime} k_{\epsilon}\right)} u_{\epsilon}^{2^{\sharp}-\epsilon} d v_{g}+\epsilon\left(R^{\prime}\right)+o(1) .
$$

If $B_{g}\left(y_{\epsilon}, \hat{k}_{\epsilon}\right) \cap B_{g}\left(x_{\epsilon}, R^{\prime} k_{\epsilon}\right) \neq \emptyset$, then

$$
\inf _{i=1, \ldots, p} d_{g}\left(y_{\epsilon}, x_{\epsilon, i}\right) \leq \hat{k}_{\epsilon}+R^{\prime} k_{\epsilon} .
$$

With (16) and (17), we then obtain that $\hat{k}_{\epsilon}=o\left(k_{\epsilon}\right)$ and $\frac{d_{g}\left(y_{\epsilon}, x_{\epsilon}\right)}{k_{\epsilon}}$ is bounded. Now we write $y_{\epsilon}=\exp _{x_{\epsilon}}\left(k_{\epsilon} \hat{y}_{\epsilon}\right)$ where $\hat{y}_{\epsilon}$ is bounded. There exists $C_{0}>0$ such that

$$
\frac{1}{k_{\epsilon}} \exp _{x_{\epsilon}}^{-1}\left(B_{g}\left(\exp _{x_{\epsilon}}\left(k_{\epsilon} \hat{y}_{\epsilon}\right), \hat{k}_{\epsilon}\right)\right) \subset B_{\xi}\left(\hat{y}_{\epsilon}, C_{0} \frac{\hat{k}_{\epsilon}}{k_{\epsilon}}\right) \text {. }
$$

We thus obtain that

$$
\int_{B_{g}\left(y_{\epsilon}, \hat{k}_{\epsilon}\right) \cap B_{g}\left(x_{\epsilon}, R^{\prime} k_{\epsilon}\right)} u_{\epsilon}^{2^{\sharp}-\epsilon} d v_{g} \leq \mu_{\epsilon}^{-\epsilon \frac{(n-4)^{2}}{8}} \int_{B_{\xi}\left(\hat{y}_{\epsilon}, C_{0} \frac{\hat{k}_{\epsilon}}{k_{\epsilon}}\right)} v_{\epsilon}^{2^{\sharp}-\epsilon} d v_{g_{\epsilon}}=o(1)
$$

since $\hat{k}_{\epsilon}=o\left(k_{\epsilon}\right)$ and $\left(v_{\epsilon}\right)$ is bounded. As a consequence,

$$
\int_{B_{g}\left(y_{\epsilon}, \hat{k}_{\epsilon}\right)} u_{\epsilon}^{2^{\sharp}-\epsilon} d v_{g} \leq \epsilon\left(R^{\prime}\right)+o(1)
$$

for all $R^{\prime}>0$. We then get that $\int_{B_{\xi}(0,1)} \hat{v}^{2^{\sharp}} d v_{\xi}=0$, a contradiction since $\hat{v}(0)=1$. This proves (13). Given an open subset $\Omega \subset \subset M \backslash O_{G}\left(x_{1}\right)$, we now get by classical regularity theorems (see for instance $[\mathrm{GT}]$ ) that $\left(u_{\epsilon}\right)$ is bounded in $C^{4, \beta}(\Omega)$. Since $u_{\epsilon}$ goes to 0 almost everywhere, it follows that

$$
u_{\epsilon} \rightarrow 0 \text { in } C^{4}(\Omega)
$$

as $\epsilon \rightarrow 0$, a relation we use in the following section. 


\section{THE CASE OF THE SPHERE}

Let $x_{0} \in \mathbb{S}^{n}$. For $\beta>1$, define

$$
u_{x_{0}, \beta}(x)=(\beta-\cos r)^{-\frac{n-4}{2}} \text { and } \tilde{u}_{x_{0}, \beta}=\left(\beta^{2}-1\right)^{\frac{n-4}{4}} u_{x_{0}, \beta}
$$

where $r=d_{h}\left(x_{0}, x\right)$. Then,

$$
P_{h}^{n}\left(\tilde{u}_{x_{0}, \beta}\right)=d_{n} \tilde{u}_{x_{0}, \beta}^{2^{\sharp}} \text { and } \int_{\mathbb{S}^{n}} \tilde{u}_{x_{0}, \beta}^{2^{\sharp}} d v_{h}=\omega_{n}
$$

where $\omega_{n}$ is the volume of the unit $n$-sphere. We now make these functions $G$-invariant. Let $x_{1} \in M$ be a point of finite orbit $O_{G}\left(x_{1}\right)=\left\{x_{1}, \ldots, x_{m}\right\}$. We define $u_{i \beta}=u_{x_{i}, \beta}, \tilde{u}_{i \beta}=\tilde{u}_{x_{i}, \beta}$ and $\tilde{u}_{\beta}=\sum_{i=1}^{m} \tilde{u}_{i \beta}$ (this function is $G$-invariant). Computing $\int_{\mathbb{S}^{n}} P_{h}^{n} \tilde{u}_{\beta} \tilde{u}_{\beta} d v_{h}$ and $\int_{\mathbb{S}^{n}} f\left|\tilde{u}_{\beta}\right|^{2^{\sharp}} d v_{h}$ we find that

$$
\int_{\mathbb{S}^{n}} P_{h}^{n} \tilde{u}_{\beta} \tilde{u}_{\beta} d v_{h}=m d_{n} \omega_{n}+d_{n} \alpha(\beta-1)^{\frac{n-4}{2}}+o\left((\beta-1)^{\frac{n-4}{2}}\right)
$$

where

$$
\alpha=\sum_{i \neq j}\left(1-\cos d_{h}\left(x_{i}, x_{j}\right)\right)^{-\frac{n-4}{2}} \omega_{n-1} \int_{0}^{+\infty} \frac{2^{n} r^{n-1}}{\left(1+r^{2}\right)^{\frac{n+4}{2}}} d r>0
$$

since $\left|O_{G}\left(x_{1}\right)\right| \geq 2$, and

$$
\begin{aligned}
& \left(\int_{\mathbb{S}^{n}} f(x) \tilde{u}_{\beta}^{2^{\sharp}} d v_{h}\right)^{\frac{2}{2^{\sharp}}} \geq f\left(x_{1}\right)^{\frac{2}{2^{\sharp}}}\left(m \omega_{n}\right)^{\frac{2}{2^{\sharp}}}\left(1+\frac{2 \alpha}{m \omega_{n}}(\beta-1)^{\frac{n-4}{2}}\right. \\
& \left.+o\left((\beta-1)^{\frac{n-4}{2}}\right)\right)
\end{aligned}
$$

provided that $\nabla^{k} f\left(x_{1}\right)=0$, for all $k=1, \ldots, n-4$. We now write that

$$
\frac{\int_{\mathbb{S}^{n}} P_{h}^{n} \tilde{u}_{\beta} \tilde{u}_{\beta} d v_{h}}{\left(\int_{\mathbb{S}^{n}} f(x) \tilde{u}_{\beta}^{\sharp \sharp} d v_{h}\right)^{\frac{2}{2^{\sharp}}}} \leq \frac{m^{\frac{4}{n}} d_{n} \omega_{n}^{\frac{4}{n}}}{f\left(x_{1}\right)^{\frac{2}{2^{\sharp}}}}\left(1-\frac{\alpha}{m \omega_{n}}(\beta-1)^{\frac{n-4}{2}}+o\left((\beta-1)^{\frac{n-4}{2}}\right)\right) .
$$

Since $d_{n} \omega_{n}^{\frac{4}{n}}=1 / K_{0}$ (see [EFJ]), it follows that

$$
\frac{\int_{\mathbb{S}^{n}} P_{h}^{n} \tilde{u}_{\beta} \tilde{u}_{\beta} d v_{h}}{\left(\int_{\mathbb{S}^{n}} f(x) \tilde{u}_{\beta}^{2^{\sharp}} d v_{h}\right)^{\frac{2}{2 \sharp}}} \leq \frac{\left|O_{G}\left(x_{1}\right)\right|^{\frac{4}{n}}}{f\left(x_{1}\right)^{\frac{2}{2^{\sharp}}} K_{0}}\left(1-\frac{\alpha}{m \omega_{n}}(\beta-1)^{\frac{n-4}{2}}+o\left((\beta-1)^{\frac{n-4}{2}}\right)\right) .
$$

Noting that $\alpha>0$, we get that

$$
\lambda^{G}(f)<\frac{\left|O_{G}\left(x_{1}\right)\right|^{\frac{4}{n}}}{f\left(x_{1}\right)^{\frac{2}{2 \sharp}} K_{0}}
$$

for all $x_{1} \in \mathbb{S}^{n}$ such that $\nabla^{k} f\left(x_{1}\right)=0$ for all $k=1, \ldots, n-4$. It then follows from Theorem 2 that the following theorem holds:

Theorem 3. Let $G$ be a compact subgroup of Isom ${ }_{g}\left(\mathbb{S}^{n}\right), n \geq 5$, acting without fixed point. Let $f \in C^{\infty}\left(\mathbb{S}^{n}\right)$ be a positive $G$-invariant function, and let $x_{0} \in \mathbb{S}^{n}$ be such that for any $x \in \mathbb{S}^{n}$,

$$
\frac{f\left(x_{0}\right)}{\left|O_{G}\left(x_{0}\right)\right|^{\frac{4}{n-4}}} \geq \frac{f(x)}{\left|O_{G}(x)\right|^{\frac{4}{n-4}}} .
$$


Assume that $\nabla^{q} f\left(x_{0}\right)=0$ for all $q=1, \ldots, n-4$. Then there exists $u \in C^{\infty}\left(\mathbb{S}^{n}\right)$, positive and $G$-invariant, such that

$$
P_{h}^{n} u=f u^{2^{\sharp}-1}
$$

and there exists a $G$-invariant conformal metric $g$ to $h$ such that $Q_{g}^{n}=f$.

We now prove Theorem 1. If there is no solution for (2), then we have (10) with a point $x_{1} \in \mathbb{S}^{n}$ such that (11) and (18) are true. Assume that we have proved that $x_{1}$ is a critical point for $f$. Since $n=5$, then (19) is true for $x_{1}$. A contradiction, and this proves the theorem. Then the proof of Theorem 1 reduces to the proof that $x_{1}$ is a critical point for $f$. We adapt an argument from Aubin. Given $(M, g)$ a compact manifold of dimension $n$, let $\left(u_{\epsilon}\right)$ be as in Lemma 1 We suppose that $\left(u_{\epsilon}\right)$ converges weakly to 0 and let $x_{1} \in M$ be such that (11) and (18) are true. With (7) we have

$$
\Delta_{g}^{2} u_{\epsilon}+\alpha \Delta_{g} u_{\epsilon}+a u_{\epsilon}=\lambda_{\epsilon}^{G}(f) f u_{\epsilon}^{2^{\sharp}-1-\epsilon} .
$$

Let $0<\delta<\min _{\substack{x, y \in O_{G}\left(x_{1}\right) \\ x \neq y}} d_{g}(x, y)$. We get with (11) that for all $z \in C^{0}(M)$,

$$
\int_{B_{g}\left(x_{1}, \delta\right)} z u_{\epsilon}^{2^{\sharp}-\epsilon} d v_{g}=\frac{z\left(x_{1}\right)}{f\left(x_{1}\right)\left|O_{G}\left(x_{1}\right)\right|}+o(1) .
$$

Now we choose $\psi \in C^{\infty}(M)$ such that Supp $\psi \subset B_{g}\left(x_{1}, \delta\right), \nabla \psi\left(x_{1}\right)=\nabla f\left(x_{1}\right)$ and $\nabla_{g}^{2} \psi\left(x_{1}\right)=0$. We then have

$$
\int_{M}(\nabla f, \nabla \psi)_{g} u_{\epsilon}^{2^{\sharp}-\epsilon} d v_{g}=\frac{|\nabla f|_{g}^{2}\left(x_{1}\right)}{f\left(x_{1}\right)\left|O_{G}\left(x_{1}\right)\right|}+o(1) .
$$

On the other hand, since $\Delta_{g} \psi\left(x_{1}\right)=0, u_{\epsilon} \rightarrow 0$ strongly in $H_{1}^{2}(M)$ and is bounded in $H_{2}^{2}(M)$, we have

$$
\begin{aligned}
& \int_{M}(\nabla f, \nabla \psi)_{g} u_{\epsilon}^{2^{\sharp}-\epsilon} d v_{g} \\
& =\int_{M}\left(\nabla\left(f u_{\epsilon}^{2^{\sharp}-\epsilon}\right), \nabla \psi\right) d v_{g} \\
& -\left(2^{\sharp}-\epsilon\right) \int_{M} f u_{\epsilon}^{2^{\sharp}-1-\epsilon}\left(\nabla u_{\epsilon}, \nabla \psi\right)_{g} d v_{g} \\
& =\int_{M} f u_{\epsilon}^{2^{\sharp}-\epsilon} \Delta_{g} \psi d v_{g} \\
& -\frac{2^{\sharp}-\epsilon}{\lambda_{\epsilon}^{G}(f)} \int_{M}\left(\Delta_{g}^{2} u_{\epsilon}+\alpha \Delta_{g} u_{\epsilon}+a u_{\epsilon}\right)\left(\nabla u_{\epsilon}, \nabla \psi\right)_{g} d v_{g} \\
& =-\frac{2^{\sharp}-\epsilon}{\lambda_{\epsilon}^{G}(f)} \int_{M} \Delta_{g}^{2} u_{\epsilon}\left(\nabla u_{\epsilon}, \nabla \psi\right)_{g} d v_{g}+o(1) \\
& =-\frac{2^{\sharp}-\epsilon}{\lambda_{\epsilon}^{G}(f)} \int_{M} \Delta_{g} u_{\epsilon} \Delta_{g}\left(\nabla u_{\epsilon}, \nabla \psi\right)_{g} d v_{g}+o(1)
\end{aligned}
$$

where we have used (20). We have

$$
\begin{aligned}
& \Delta_{g}\left(\nabla u_{\epsilon}, \nabla \psi\right)_{g}=\left(\nabla \Delta_{g} u_{\epsilon}, \nabla \psi\right)_{g} \\
& \quad+O\left(\left|\nabla u_{\epsilon}\right|_{g}\right)+O\left(|x|\left|\nabla_{g}^{2} u_{\epsilon}\right|_{g}|\nabla \psi|_{g}\right)+O\left(\left|\nabla_{g}^{2} u_{\epsilon}\right|_{g}\left|\nabla_{g}^{2} \psi\right|_{g}\right) .
\end{aligned}
$$


Then, with (18), (20) and since $\left(u_{\epsilon}\right)$ is bounded in $H_{2}^{2}(M)$, we get that

$$
\begin{aligned}
& \int_{M}(\nabla f, \nabla \psi)_{g} u_{\epsilon}^{2^{\sharp}-\epsilon} d v_{g}=-\frac{2^{\sharp}-\epsilon}{\lambda_{\epsilon}^{G}(f)} \int_{M} \Delta_{g} u_{\epsilon}\left(\nabla \Delta_{g} u_{\epsilon}, \nabla \psi\right)_{g} d v_{g}+o(1) \\
& =-\frac{2^{\sharp}-\epsilon}{2 \lambda_{\epsilon}^{G}(f)} \int_{M}\left(\nabla\left(\Delta_{g} u_{\epsilon}\right)^{2}, \nabla \psi\right)_{g} d v_{g}+o(1) \\
& =-\frac{2^{\sharp}-\epsilon}{2 \lambda_{\epsilon}^{G}(f)} \int_{M}\left(\Delta_{g} u_{\epsilon}\right)^{2} \Delta_{g} \psi d v_{g}+o(1)=o(1)
\end{aligned}
$$

since $\Delta_{g} \psi\left(x_{1}\right)=0$. Hence $\nabla f\left(x_{1}\right)=0$. Taking $M=\mathbb{S}^{n}$, this ends the proof of Theorem 1

\section{ACKNOWLEDGEMENTS}

The author thanks Olivier Druet, Emmanuel Hebey, and Antoinette Jourdain for their useful remarks on the manuscript.

\section{REFERENCES}

[Bra] Branson, T.P. Group representations arising from Lorentz conformal geometry, J. Funct. Anal., 1987, 74, 199-291. MR 90b:22016

[DHL] Djadli, Z.; Hebey, E.; Ledoux, M. Paneitz type operators and applications, Duke Math. J., 2000, 104, 129-169. MR 2002f:58061

[Dru] Druet, O. The best constants problem in Sobolev inequalities, Math. Ann., 1999, 314, 327-346. MR 2000d:58033

[EFJ] Edmunds, D.E.; Fortunato, F.; Janelli, E. Critical exponents, critical dimensions, and the biharmonic operator. Arch. Ration. Mech. Anal. 1990, 112, 269-289. MR 91k:35191

[EsSc] Escobar, J. F.; Schoen, R. M. Conformal metrics with prescribed scalar curvature, Invent. Math., 1986, 86, 243-254. MR 88b:58030

[GT] Gilbarg, D.; Trudinger, N.S. Elliptic Partial Differential Equations of Second Order, 2nd Ed., Grundlehren der Mathematischen Wissenschaften, Springer-Verlag: Berlin, 1983, Vol. 224, 513 pp. MR 86c:35035

[Heb] Hebey, E. Changements de métriques conformes sur la sphère. Le problème de Nirenberg, Bull. Sci. Math., 1990, 114, 215-242. MR 91h:53017

[HeRo] Hebey, E.; Robert, F. Coercivity and Struwe's compactness for Paneitz type operators with constant coefficients, Calc. Var. Partial Differ. Equ., 2001, 13, 491-517.

[Jou] Jourdain, A. Paneitz type operator and first spherical harmonics, Preprint 2000.

[Mos] Moser, J. On a nonlinear problem in differential geometry, Dyn. Syst. (Academic Press, New York, 1973). MR 49:4018

[Pan] Paneitz, S. A quartic conformally covariant differential operator for arbitrary pseudoRiemannian manifolds, Preprint 1983.

Département de Mathématiques-Site Saint-Martin, Université de Cergy-Pontoise, 2, Avenue Adolphe Chauvin, F 95302 Cergy-Pontoise Cedex, France

E-mail address: Frederic.Robert@math.u-cergy.fr 